Gunter Scholtz*

Bochum

\title{
Das Problem der Allgemeinbildung geschichtlich betrachtet**
}

\section{Von der egkykliospaideia zur Enzyklopädie}

Das Bildungsideal im antiken Griechenland nannte man egkykliospaideia, und das war die Erziehung freier Bürger durch mousiké, d.h. durch Musik als Verknüpfung von Rhythmus/Tanz, Melodie und Wort ${ }^{1}$. Die erste Form der egkykliospaideia bestand also gar nicht in einer intellektuellen

* Professor of History and Theory of the Human Sciences in the department of philosophy at Ruhr-Universität Bochum. Homepage: http://www.ruhr-uni-bochum.de/philosophy/ mitglieder/scholtz.html.de

** Dieser Beitrag ist der Text eines Vortrages, den ich im Juni 2013 auf Einladung der Polskie Towarzystwo Pedagogiczne in Kraków gehalten habe. Inzwischen erschien eine ausgearbeitete Fassung unter dem Titel: Das Ende der Enzyklopädie - eine Tragödie der Kultur?, „Philotheos“ 14 (2014), S. 3-16.

${ }^{1}$ Siehe dazu und zum Folgenden H. Fuchs, EnkykliosPaideia, w: Reallexikon für Antike und Christentum, Bd. 5, Stuttgart 1962, Sp. 365-398; Enzyklopädie, ebd. Sp. 504-515; J. Dolch: Lehrplan des Abendlandes. Zweieinhalb Jahrtausende seiner Geschichte, Darmstadt 1982; J. Henningsen, ,Enzyklopädie“. Zur Sprach- und Bedeutungsgeschichte eines pädagogischen Begriffs, „Archiv für Begriffsgeschichte“ 10 (1966), S. 271-362; H.-T. Johann (Hg.), Erziehung und Bildung in der heidnischen und christlichen Antike, Darmstadt 1976; U. Dierse, Enzyklopädie. Zur Geschichte eines philosophischen und wissenschaftstheoretischen Begriffs. Archiv für Begriffsgeschichte, Supplementheft 2, Bonn 1977; I. Tomkowiak (Hg.), Populäre Enzyklopädien. Von der Auswahl, Ordnung und Vermittlung des Wissens, Zürich 2002. 
Schulung oder in einer Wissensvermittlung, sondern es war eine musische Erziehung, und sie hatte nicht die Erlangung von umfänglichen Kenntnissen zum Ziel, sondern eine sittlichen Haltung, denn sie galt der sittlichen Formung des ganzen Menschen als einer leib-seelischen Einheit. Das Wort egkyklios bedeutet allgemein, gewöhnlich. Demnach war egkykliospaideia die allgemeine oder grundlegende Erziehung. Man sieht es dem Begriff und der Sache nicht an, dass daraus später die „Enzyklopädie“ wurde.

Erst in der römischen Welt wurde der alte griechische Ausdruck für die Erlangung von Kenntnissen und intellektuellen Fähigkeiten gebraucht, die man mit dem lateinischen Ausdruck orbis doctrinae oder orbis disciplinarum zusammenfasste (also Kreis der Lehre, Kreis der Disziplinen). Dieser „Kreis“ war nicht der Inbegriff allen Wissens, sondern es waren die mathematischen Disziplinen Arithmetik, Geometrie, Musik und Astronomie, die schon Platon für die Bildung zum Philosophen wichtig fand, und die sprachlichen Disziplinen Grammatik und Rhetorik, die Aristoteles als Voraussetzung weiterer Bildung empfahl. Daraus wurden dann die Artes liberales, die sogenannten freien Künste, die durch Zufügung der Dialektik auf die Zahl Sieben festgelegt wurden.

Mit dem Begriff ars, Kunst, meinte man nicht nur Kenntnisse, sondern jeweils eine Fähigkeit, die auf Talent, Übung und Wissen basiert. Im Studium einer solchen Kunst erwarb man also nicht nur Wissen, sondern lernte etwas gut und richtig zu tun, z.B. Reden zu halten. Der ganze Kreis dieser Künste war so beschaffen, dass junge Leute ihn durchlaufen konnten. Im Mittelalter galten diese Artes liberales als Grundlage für den Erwerb aller Spezialkenntnisse in Theologie, Jurisprudenz und Medizin. Sie lieferten also die Allgemeinbildung.

Erst im Renaissance-Humanismus formte man den lateinischen Ausdruck encyclopaedia, und es entstanden auch eigene Werke, die das neue Wort im Titel trugen. Sie bemühten sich, das gesamte Wissen in eine plausible Ordnung zu bringen.

Eines der ersten großen Werke ist die Encyclopaedia von J. H. Alsted, die in der Ausgabe von 1630 bereits sieben Bände mit insgesamt rund 2400 eng bedruckten Seiten umfasst. Nach Alsted enthält eine Enzyklopädie alles, was überhaupt würdig ist, gelehrt zu werden. Sein Werk hat noch immer einen didaktischen Charakter. Es beansprucht, all das zusammenzufassen, was ein Mensch in seinem Leben lernen kann und was ein Universal-Gelehrter sich auch aneignen muss. Die philosophische Systematik ist zugleich pädagogisch gemeint, denn die Ordnung des Wissens soll das methodische Studium erleichtern: Das Werk beginnt, in dem es auf 26 Seiten 38 Tafeln bietet, 
auf denen alle Disziplinen geordnet aufgelistet werden. Noch immer ist also die Methode - also der methodos, der Weg - der Einteilung im Hinblick auf das methodische Studium entworfen. Der verfügbare Stoff aber ist schon so groß, dass Alsted bei Darstellung der Disziplinen sich jeweils nur auf die „Prinzipien“" konzentriert.

$\mathrm{Zu}$ dieser Begrenzung ermahnte im 17. Jahrhundert dann besonders Comenius: Eine Enzyklopädie, lehrte Komenský, soll nicht alles, sondern das Ganze zeigen (gr. pan), es soll eine „Pansophie“ sein. Nicht der Materialreichtum soll an erster Stelle stehen, da er die menschliche Fassungskraft überfordern kann, sondern die Ordnung, die Systematik. Eine Enzyklopädie soll wie ein klug strukturiertes Gebäude oder wie ein Baum sein, dessen Zweige aus einem Stamm und aus einer Wurzel herauswachsen. Comenius verbindet die Didaktik mit der Metaphysik: Nur wenn ein überschaubares Ganzes dargeboten wird, kann diese Totalität des Wissens studiert und in ihm auch die Schönheit der Schöpfung erkannt werden.

Der Philosoph Leibniz teilt um 1700 diese metaphysische Auffassung und die Forderung nach einer überzeugenden Ordnung. Aber er bemüht sich in seinen Überlegungen zur Enzyklopädie um eine rationale Systematik alles dessen, was überhaupt gewusst werden kann. Deshalb richtet sich sein Projektentwurf wesentlich an die Gelehrten, an die Forschergemeinschaft. Hier ist vorausgesetzt, dass eine Enzyklopädie nicht die Sache eines einzigen Verfassers sein kann und dass der Inhalt auch in keinem einzigen menschlichen Kopf Platz findet.

In der großen französischen Enzyklopädie, die von 1751 bis 1772 erschien, ist das bereits vorausgesetzt, und sie bedeutet einen Umbruch. Verfasst von einem Autorenkollektiv von gut 170 Mitarbeitern, kann und soll das Werk mit seinen 28 Bänden und fast 72.000 Artikeln von keinem einzelnen Leser durchgelesen und studiert werden. Wie der Titel Encyclopédie zeigt, wurde der Anspruch auf eine kohärente, systematische Ordnung des Wissens beibehalten, und diese wird aus dem menschlichen Erkenntnisvermögen entwickelt. Aber diese Ordnung des Wissens erscheint nur in einer eigenen Tafel, in einem gesonderten Teil des Werkes. Die Fülle des Stoffes aber wird dem Benutzer nun in alphabetischer Folge geboten, und insofern ist das Werk ein Dictionnaire und hat die Gestalt eines Lexikons oder Nachschlagewerkes. Das Systemganze hat sich von der Summe der Teile, vom Gesamt des Wissens, abgelöst. Das Wissen hat bereits einen so großen Umfang angenommen, dass auch die einzelnen Artikel hier nur als Landkarten verstanden werden, die nur über ein bestimmtes Gebiet orientieren, ohne das Land selbst ganz zeigen zu wollen. Wie der Herausgeber Diderot schreibt, 
soll das Werk der Bildung, ja sogar dem Glück und der Tugend dienen. Aber nirgends wird gesagt, welche Artikel dafür studiert werden müssen. Vielmehr wird nun ein Benutzer vorausgesetzt, der selbst frei auswählt, was er wissen möchte. Man geht davon aus, dass das Wissen immer der Bildung, dem Glück und der Tugend dient. Das frühere Ziel, dem Einzelnen den wichtigsten Kreis der Grundkenntnisse zu bieten, wurde durch die Absicht ersetzt, durch Vermittlung von allem verfügbaren Wissen die Ausbreitung der Aufklärung und mit ihr die Reform der Gesellschaft zu fördern. Der Adressat ist nun der ganze gebildete Bürgerstand, es sind nicht nur die Studierenden oder die Gelehrten.

Seit dem 18. Jahrhundert haben sich dann im Wesentlichen drei Formen von Enzyklopädien herausgebildet:

(1) Die allgemeinen Enzyklopädien eröffnen in alphabetischer Anordnung einen Zugang zu allen Wissensbereichen. Sie wollen erreichtes Wissen sammeln, das als wichtig angesehen wird, und einem möglichst großen Leserkreis leicht zugänglich machen. Alle Nationen haben seit dem 19. Jahrhundert solche Werke geschaffen, besonders berühmt wurde die Encyclopaedia Britannica (die erstmals 1768-1771 in drei Bänden erschien). „Enzyklopädisch" heißt hier: umfassend, und die Qualität dieser Werke besteht in ihrem Materialreichtum. Deshalb macht es sachlich keinen Unterschied, ob diese Werke „Enzyklopädie“ oder „Universallexikon“ heißen - wie schon das Grosse vollständige Universal-Lexicon Aller Wissenschaften und Künste, das J. H. Zedler mit seinen 68 umfangreichen Bänden in den Jahren 1732 bis 1754 herausgab.

(2) Sodann erschienen philosophische Enzyklopädien und Literatur zur Enzyklopädie als System der Wissenschaften. „Enzyklopädisch“ meint hier: geordnet, systematisch, einen Überblick auf das gegliederte Ganze des Wissens gewährend. In diesen Werken geht es zentral um Fragen der Klassifikation der Wissenschaften, die in jenen Universallexika fehlen. In diesen Kontext gehört aus dem frühen 19. Jahrhundert Hegels Enzyklopädie der philosophischen Wissenschaften, aber auch August Comtes Ausführungen zum ,enzyklopädischen Gesetz“ der positiven Wissenschaften. Solche Werke sind auf ein „System“ ausgerichtet, das den Wissenschaften ein Fundament und eine Ordnung geben will.

(3) Daneben wurden Spezialenzyklopädien zu den einzelnen Zweigen der Wissenschaften ediert. Das waren zunächst, seit der Mitte des 18. Jahrhunderts, Einleitungsvorlesungen, die den Studierenden einen Überblick über ihr Fachgebiet ermöglichen sollten, später aber auch lexikalische Nachschlagewerke. 
In allen drei Ausrichtungen finden wir noch immer didaktische Aspekte, aber diese sind nicht dominant. Die allgemeinen Enzyklopädien wollen das von Experten erarbeitete Wissen einem möglichst großen Leserkreis zugänglich und verständlich machen. Die didaktische Leistung der Autoren besteht hier z.B. darin, die wissenschaftliche Terminologie eines speziellen Faches möglichst in allgemeine Sprache zu übersetzen und auf knappem Raum die wichtigsten Informationen zu geben.

Die philosophischen Enzyklopädien wie die Hegelsche haben zumindest einen Bezug zur Bildung. Der menschliche Geist soll lernen, alles im Kontext zu sehen, und er soll sich nicht in der Fülle des Einzelwissens verlieren, sondern seine Einheit in der Mannigfaltigkeit des Wissens behaupten und wiederfinden. Die Einheit des Systems zielt also auf die Einheit des Geistes.

Nur der Positivist August Comtes verfolgte mit seinem ,enzyklopädischen Gesetz", das den Zusammenhang der Wissenschaften zeigt, ausdrücklich ein pädagogisches Ziel, und sogar ein sehr hochgestecktes: Er wollte die Menschheit lehren, mit Hilfe der sogenannten positiven Wissenschaften, also der Naturwissenschaften und der Soziologie, zu einem befriedeten Gesellschaftszustand fortzuschreiten. Eine gewisse Ähnlichkeit dazu hat dann am Beginn des 20. Jahrhunderts auch das Programm einer Enzyklopädie von Otto Neurath aus dem Kreis der Wiener Neopositivisten: Alles wirkliche Wissen soll in exakte Einheitssprache überführt werden, damit es den Massender Menschen Nutzen bringe. - Wir zögern, für solche Menschheitsbeglückung durch exakte Wissenschaft das Wort „Bildung“ einzusetzen, da hier der Einzelne völlig gleichgültig geworden ist und letztlich nur die Wissenschaft herrschen soll.

Die allgemeinen enzyklopädischen Nachschlagewerke und Universallexika wurden seit dem 18. Jahrhundert immer von sehr vielen Autoren verfasst. Die Systeme aber waren Werke bestimmter Autoren wie Hegel oder Comte. Die Einheit oder der Zusammenhang des Wissens war also die Leistung bestimmter Individuen, sie resultierte nicht aus der Entwicklung der Wissenschaften selbst. Lieferten die allgemeinen Enzyklopädien eine Mannigfaltigkeit ohne Einheit, so konstruierten die philosophischen Enzyklopädien Einheitssysteme, ohne die tatsächliche Mannigfaltigkeit der fortschreitenden Wissenschaften berücksichtigen zu können. Comte klammerte sogar alle Geistes- oder Kulturwissenschaften aus.

Inzwischen ist das Wort „Enzyklopädie“ problematisch geworden. Der Ausdruck verspricht offensichtlich mehr, als man heute noch bieten kann. Die allgemeinen Enzyklopädien veralten immer schneller durch den Wissenszuwachs, so dass sie vermutlich bald nur noch - wie wikipedia - in di- 
gitaler Form erscheinen, um ständig leicht korrigiert und erweitert werden zu können. In Deutschland hat das große Brockhaus-Lexikon den Titel „Enzyklopädie“ inzwischen schon aufgegeben. Eine Enzyklopädie der philosophischen Wissenschaften ist so wenig in Sicht, wie längst die Philosophie ihren Systemcharakter verlor und nicht mehr beanspruchen kann, alle Wissenschaften grundzulegen. Dafür wächst die Literatur zu Geschichte von Begriff und „Problem“ der Enzyklopädie. So ist zunächst die pädagogische Bedeutung des Begriffs immer mehr geschrumpft, und jetzt scheint der Begriff bald ganz von der Bühne abzutreten, so wie es ja längst auch keine „Universalgelehrten“ mehr gibt. Ein abgeschlossenes „Werk“, welches allem Wissen Rechnung trägt, wurde durch das beschleunigte Wachstum des Wissens ebenso unmöglich wie eine verbindliche Ordnung und ein Überblick über alle Wissensgebiete. Worin könnte in dieser Situation eine egkykliospaideia im Sinne der Artes liberales, worin könnte „Allgemeinbildung“ bestehen?

\section{Georg Simmels Diagnose}

Für das Verständnis und die Beurteilung der entstandenen Situation scheint mir die Kulturphilosophie Georg Simmels nützlich zu sein. Der Soziologe und Philosoph Simmel, der von 1858 bis 1918 lebte, gehört zu den interessantesten Gestalten am Übergang zum 20. Jahrhundert. Um den Begriff der Kultur zu bestimmen, unterscheidet Simmel zuerst in traditioneller Weise Kultur und Natur, entdeckt dann aber einen zweiten Gegensatz im Bereich der Kultur selbst, nämlich zwischen der „subjektiven Kultur“, der Kultivierung oder Bildung der Einzelnen, und der „objektiven Kultur“ einer

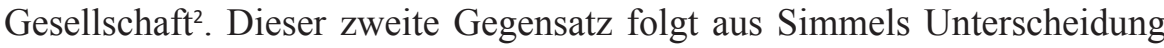
zwischen der subjektiven Seele mit ihren Anlagen, Strebungenund Interessen auf der einen Seite und den kollektiv hervorgebrachten geistigen Gebilden und Systemen wie Religion, Kunst, Recht, Wirtschaft, Technik usw. auf der anderen Seite. Während das Leben der subjektiven Seele begrenzt ist und sich im Fluss der Zeit stets verändert, erlangen jene Produkte des Menschen

${ }^{2}$ Georg Simmel hat mehrere Abhandlungen zur Bestimmung der Kultur verfasst: Persönliche und sachliche Kultur (1900), Gesamtausgabe, hg. von OttheinRammstedt, Frankfurt a.M. 1989 ff, Bd. 5, S. 560-582; Vom Wesen der Kultur (1908), Ebd. Bd. 8, S. 363-373; Der Begriff und die Tragödie der Kultur (1911), Ebd. Bd. 14, S. 385-416; Die Krisis der Kultur (1916), Ebd. Bd. 13, S. 190-201; Wandel der Kulturformen (1916), Ebd. S. 217-223; Der Konflikt der modernen Kultur (1918), Ebd. Bd. 16, S. 181-207 [wyd. polskie Filozofia kultury. Wybór esejów, tłum. W. Kunicki, Wydawnictwo Uniwersytetu Jagiellońskiego, Kraków 2007]. 
eine gewisse Konstanz und Festigkeit, und sie sind in ständigem Wachsen begriffen. Die Kultursysteme erhalten und entwickeln sich nur durch die produktive Tätigkeit der Individuen, und die subjektive Seele benötigt die Systeme der „objektiven Kultur“, um ihre individuellen Anlagen entfalten zu können. Beide Seiten verhalten sich wie die Sprache und die Sprecher, sie sind auf einander angewiesen. Der Einzelne erreicht nur mit Hilfe der objektiven Kultur seine ,subjektive Kultur", d.h. die Kultivierung seiner Person und seine Bildung. Kultivierung ist der Weg der Seele zu sich selbst, sagt Simmel, und dieser Weg ist immer ein Umweg: Die Seele als noch nicht entfaltete Einheit öffnet sich den vielfältigen Gestaltungen der objektiven Kultur, verarbeitet sie und integriert sie zu einer entfalteten, inhaltsreichen Einheit.

Nur wenn die Kultivierung der Einzelnen gelingt, spricht Simmel von „Kultur“ im vollen Sinne des Wortes, Kultur ist für ihn wesentlich die Bildung der Einzelnen. Diese Bildung aber sieht erwachsend gefährdet oder sogar untergraben: Während nämlich die objektive Kultur ständig wächst, löst sie sich von den Einzelnen ab, ja sie tritt ihnen mehr und mehr als etwas Fremdes und Undurchschaubares entgegen, so dass die Vermittlung misslingt und die individuelle Kultivierung oder Bildung schrumpft. Die kulturelle Evolution der Gesellschaft und die Kultivierung der Einzelnen nehmen verschiedene Wege. In Hegels Sprache ausgedrückt: Der individuelle Geist kehrt aus seiner Entfremdung nicht mehr zu sich selbst zurück. Mit der kulturellen Entwicklung und dem wachsenden Reichtum an Kultur der Gesellschaft ist so für Simmel eine Verarmung des kultivierten Lebens der Einzelnen verbunden.

Der wichtigste Bereich, an dem Simmel seine Auffassung gewonnen hat, ist das Geld - zur Philosophie des Geldes publizierte er 1900 ein eigenes umfängliches Werk ${ }^{3}$. Die Erfindung des Geldes ist für Simmel eine der bedeutsamsten Fortschritte der menschlichen Kultur. Es erleichtert erheblich den Austausch von Gütern und dient damit der Befriedigung der Bedürfnisse, es beendet den Erwerb durch Raub und Plünderung und fördert auch die individuelle Freiheit. Denn es bietet die Möglichkeiten für eine vielfältige Wahl von Entscheidungen und eröffnet den Zugang zu vielerlei Gütern, die zum Verkauf stehen. Im Verlauf des gesellschaftlichen Zusammenlebens aber verselbständigt sich das Geld, es gewinnt eine Eigendynamik und nimmt schließlich die Menschen in ihren Dienst. Es wird nicht mehr als Mittel für

${ }^{3}$ G. Simmel, Die Philosophie des Geldes, hg. von David P. Frisby, Klaus Christian Köhnke, Gesamtausgabe Bd. 6, Frankfurt a. M. 1989. 
wichtige Zwecke, sondern als Selbstzweck erstrebt, drängt die wirklichen Zwecke des Menschen in den Hintergrund und lässt die Menschen seelisch verarmen. An der Börse bekommt die Bewegung des Finanzmarktes den Charakter einer Naturgesetzlichkeit. Das Geld kann nun die Freiheit und die Befriedigung der Bedürfnisse auch gefährden. Simmel schrieb das, obwohl er noch keine Weltwirtschaftskrise erlebt hatte.

Besonders auch an der Technik verdeutlicht Simmel, dass Mittel zu Zwecken werden. Die Maschinen werden zwar immer vollkommener und schließlich intelligenter als der Mensch. Aber erstens weiß kein Einzelner mehr das technische Produkt als sein eigenes Werk, ja es wird ihm als Ganzes oft unbegreiflich, da er immer nur an Teilbereichen arbeitet. Und zweitens dienen die technisch perfekten Geräte nicht mehr immer den menschlichen Bedürfnissen und schon gar nicht unserer eigenen Vervollkommnung. Daraus resultiert ein Gefühl der Unzufriedenheit und des Missbehagens, das für den modernen Menschen typisch ist. In der Tat: Unsere Drucker und die Rechtschreibprogramme werden immer besser - unsere Handschriften und unsere Schreibkultur immer schlechter.

Man kann Simmels Lebensphilosophie und seiner idealistischen Orientierung an einem Subjekt, das durch Bildung zu einer höheren, entfalteten Einheit kommt, skeptisch gegenüber stehen. Man muss auch die Trennung von subjektiver und objektiver Kultur nicht wie Simmel eine "Tragödie“ nennen. Aber man wird schwerlich leugnen können, dass sich in der Entwicklung der Zivilisation anonyme Prozesse vollziehen, die sich vom Willen der Einzelnen abgelöst haben, die niemand verantwortet und niemand steuert und die der Kultivierung der individuellen Menschen nicht immer dienlich sind.

Gerade auch im Bereich des Wissens lässt sich Simmels These belegen. Der Historiker Alfred Heuß sagte, die intensive Arbeit in der Geschichtswissenschaft und der Mangel an historischem Bewusstsein in der Bevölkerung seien nur zwei Seiten desselben Vorgangs: Da sich alle historischen Vorgänge durch die Forschung als hoch kompliziert erweisen, nehme man Abstand von dieser verwirrender Informationsfülle.

Schon an der Entwicklung des Begriffs der Wissenschaft lässt sich zeigen, was Simmel meinte. Bei Platon und Aristoteles war episteme (Wissen) eine Tugend und als solche eine hexis, eine Haltung von Menschen. Noch im 18. Jahrhundert wurde scientia (Wissen/Wissenschaft) als eine „Fertigkeit des Verstandes“ betrachtet und als ein Habitus von konkreten Menschen. Auch das deutsche Wort „Wissenschaft" bezeichnete damals noch etwas, was man hatte, wie man eine Tugend hat. Bald aber wurde Wissenschaft 
etwas, was kein Einzelner mehr haben kann, sie wurde ein anonymes Kollektivunternehmen, dem der Einzelne nur noch dient. Wissenschaft und Wissen trennten sich. Die Schrumpfung der subjektiven Kultur zeigt sich hier daran, dass im Unterschied zur traditionellen Philosophie heute niemand mehr genau weiß, was „Wissen“ überhaupt ist. Ein Blick in die neuen Handbücher der Wissenschaftstheorie verrät uns, dass man der Frage nach dem Wissen lieber aus dem Wege geht und nur zu bestimmen sucht, was „Wissenschaft" ist.

Was unsere Skizze des Weges der egkykliospaideia zu den neueren Enzyklopädien zeigte, fügt sich diesen Befunden gut ein. Denn zunächst bezeichnete der Ausdruck die seelisch-sittliche Bildung freier Bürger und dann den Inbegriff der Grundkenntnisse, die ein Rhetor oder Theologe haben musste. Daraus wurde in der Neuzeit die Enzyklopädie als systematischer Überblick über alle Wissensbereiche, der von den Gelehrten studiert und angeeignet werden sollte, besonders dann, wenn sie „Universalgelehrte“ werden wollten. Schließlich enthielten die Enzyklopädien das Wichtigste von allem verfügbaren Wissen und lösten sich wachsend von didaktischen Zielsetzungen $\mathrm{ab}$, und niemand konnte mehr beanspruchen, ein Universalgelehrter zu sein. Nun entstand die Frage, was von den Mengen an Wissen für den Einzelnen wichtig ist, ja was „Bildung“ angesichts der Wissensmengen überhaupt sein könnte. Mit Anwachsen der Wissenschaften, der objektiven Kultur, wie Simmel sagte, wird also die subjektive Kultur, die Kultivierung oder Bildung, problematisch.

\section{Lösungsversuche}

Weil für Simmel jener Entfremdungsprozess zwischen dem objektiven Geist und den Belangen des Einzelnen unvermeidbar war, nannte er das Ergebnis seiner Beobachtungen „die Tragödie der Kultur". Aber bis heute wollte sich kaum jemand mit dieser Tragödie abfinden. Man kann vermutlich die gesamte Geschichte der neueren Bildungstheorien als Geschichte der Antworten auf das von Simmel scharf benannte Problem rekonstruieren.

Es ließe sich m.E. gut zeigen, wie die von Simmel beschriebene Situation der modernen Kultur die politischen Großideologien begünstigte, die - nur auf bestimmte kollektive Ziele ausgerichtet - den Reichtum der objektiven Kultur in ihrem Sinne sogleich reduzieren und den Einzelnen in ihre Dienste einspannen. Aber als seriöse typische Reaktionen kann man Existentialismus und Szientismus betrachten, die von verschiedener Seite das ganze Denkmo- 
dell Simmels destruieren. Sie entstanden schon in den 20er Jahren, aber ihre Grundrichtung blieb erhalten: Die Existenzphilosophie kehrt im Namen der subjektiven Freiheit der objektiven Kultur zumeist den Rücken zu, und für den Szientismus (etwa des Wiener Kreises) gibt es nur objektive Kultur, und von ihr nur eine Form: die exakte Wissenschaft. Bei beiden fiel die Kultivierung oder die individuelle Bildung (als Verbindung von Subjektivität und objektivem Geist) aus ihrem Gesichtsfeld heraus. Deshalb hebe ich kurz zwei andere Konzeptionen hervor, die wirklich auf Bildung ausgerichtet waren.

(1) In Wilhelm von Humboldts frühen Gedanken zur Bildung um 1800 dominierte zunächst die Orientierung an der griechisch-antiken Kultur ${ }^{4}$. Sie schien ihm alles vorbildlich zu enthalten, was der Moderne fehlt: eine Verschmelzung von Verstand und Sinnlichkeit, von natürlicher und geistiger Schönheit; Religion und Moral waren laut Humboldt keine Geistesfesseln und übten keine Herrschaft über die Gesinnung aus, sondern waren von Liberalität und Schönheit geprägt. Wer diese Kultur studiere, erreiche den „edelsten Zweck“, nämlich die harmonische Ausbildung aller Kräfte des Menschen. Denn wer diese vergangene Kultur verstehen wolle, der müsse sich ihr ähnlich machen (gemäß dem alten Prinzip: Gleiches wird nur von Gleichem erkannt). Deshalb war für Humboldt die wissenschaftliche, nämlich historisch-philologische Beschäftigung mit der griechischen Kunst- und Gedankenwelt zugleich die Aneignung eines Kulturideals: „Die Griechen [...] sind für uns, was ihre Götter für sie waren" "5. Wer also die alte Kultur studiert, der erfährt, was für den Menschen möglich, erstrebenswert und sinnvoll ist. Als Reaktion auf das Anwachsen des Wissens vollzieht Humboldt damit eine Reduktion auf das, was ihm wichtig erscheint, auf die Quellen der europäischen Kultur. Mit Simmel gesagt: Die Bildung des Einzelnen soll in der Moderne erreicht werden auf dem Umweg über das Studium einer objektiven Kultur der Vergangenheit, der griechischen Antike. - Aber der moderne Mensch kann letztlich kein antiker Grieche werden, und Humboldts Bild der Antike war auch einseitig und stark idealisiert.

Der spätere Humboldt hatte eine andere Hoffnung, Bildung im Zeichen der sich ausbreitenden Wissenschaften zu erreichen: durch die Verbindung

4 Siehe Humboldts Abhandlungen: Über das Studium des Alterthums, und des griechischen insbesondere (1793), Werke in fünf Bänden, hg. von Andreas Flitner, Klaus Giel, Bd. 2, Darmstadt ${ }^{2} 1969$, S. 1-24; Latium und Hellas oder Betrachtungen über das klassische Alterthum (1806), ebd. S. 25-64; Ueber den Charakter der Griechen, die idealische und historische Ansicht desselben, ebd. S. 65-72.

${ }^{5}$ W. von Humboldt, Ueber den Charakter der Griechen, S. 65. 
von Philosophie und empirischer Forschung zu einer wissenschaftlichen Forschung in philosophischem Geist. Was er damit meinte, geht aus seinen Schriften zur Sprachphilosophie hervor. Die vergleichende Sprachwissenschaft führe zur Einsicht, dass die Menschheit eine in Sprach-Individualitäten gegliederte, aber zusammenhängende Familie ist. Das Sprachstudium zeige sowohl Zusammengehörigkeit als auch die Individualität der Menschen und sei für ihn deshalb zugleich ein Studium der Humanität. Mit Simmel gesprochen: Die eigene Bildung wird beim Sprachstudium nicht durch Informationslast bedroht, sondern gefördert, weil man während des Studiums eigentlich schon immer am Ziel ist, bei der Idee der Humanität, die sich nur immer deutlicher herausschält. Humboldt konnte auf den philosophischen Geist zählen, weil dieser Geist in seiner Zeit noch hinreichend kultiviert wurde. Aber dass seine Konzeption nicht sehr stabil war, sieht man daran, dass die moderne Sprachwissenschaft sich nicht mehr als Humanitätsstudium versteht.

(2) Humboldt arbeitete eng mit dem Theologen und Philosophen Friedrich Schleiermacher zusammen, in dem manche heute den Begründer einer wissenschaftlichen Pädagogik sehen ${ }^{6}$. Auch Schleiermacher verlangte eine Verbindung von Philosophie und Einzelforschung. Aber zu diesem Zweck sollte besonders in den Universitäten das Fach Philosophie gelehrt werden, und er entwickelte auch selbst ein eigenes Ordnungssystem der Wissenschaften - allerdings eines, das für den Fortgang des Wissens offen ist.

Wenn er besonders der Universität die Aufgabe zuwies, den Studierenden in Form einer „Enzyklopädie“ einen Überblick zu verschaffen, so verlangte er damals mehr, als für uns heute vermutlich einlösbar ist. Aber wir entsprechen heute seinem Gedanken, wenn man in allen Disziplinen auch Überblicksvorlesungen sowie ein Studium Generale anbietet, wie man das bei uns nennt, und wenn man spezielle Forschungsfragen in einen größeren Kontext einstellt. Die sich spezialisierenden Wissenschaften verlangen jedenfalls noch immer Ergänzungen, Kompensationen ihrer Einseitigkeit.

Die Bildungseinrichtungen aber müssen laut Schleiermacher vor allem mit den verschiedenen Sphären der gegenwärtigen Kultur vertraut machen, die für ihn zugleich Sozialsphären oder „Gemeinschaften“ sind, nämlich Kommunikationsgemeinschaften mit eigenen Kriterien. Solche Kultur- und Sozialsphären sind bei Schleiermacher Wissenschaft, Religion/Kunst, Politik/

${ }^{6}$ F. Schleiermacher, Texte zur Pädagogik. Kommentierte Studienausgabe in zwei Bänden, hg. von Jens Brachmann und Michael Winkler, Frankfurt 2000. 
Staat, freie Geselligkeit/Privatsphäre einschließlich Familie ${ }^{7}$. Die Entwicklung aller Kräfte des Menschen, die auch Humboldt forderte, realisiert sich demnach durch Teilnahme an verschiedenen Kulturbereichen. Ob Schleiermachers Systematik dieser Sphären heute noch ausreicht, lasse ich dahingestellt. Aber seine Konzeption trägt der historisch erfolgten Differenzierung der Kultur Rechnung und anerkennt verschiedene „Wertsphären“, wie der Neukantianismus sagte, oder verschiedene „Kommunikation-Codes“, wie es die Systemtheorie heute ausdrückt. Das hat den Vorteil, dass nicht alles mit demselben politischen, ökonomischen oder religiösen Maß gemessen wird. Michael Walzers Buch über die „Sphären der Gerechtigkeit“" verfolgt heute einen ähnlichen Gedanken ${ }^{8}$. In unserer Gegenwart, in der das ökonomische Denken alle Bereiche der Gesellschaft zu beherrschen tendiert, scheint mir die Erinnerung daran, dass es nicht nur das Kriterium „rentabel/unrentabel“ gibt und geben sollte, von größter Wichtigkeit zu sein.

Simmel sah besonders in der Philosophie und in der Kunst Heilmittel angesichts der Verselbständigung der objektiven Kultur. Im Philosophieren und künstlerischen Gestalten verliere sich der Einzelne nicht, gebe nicht seine innersten Interessen auf, sondern realisiere seine ihm wichtigen Zwecke. Das hätte Schleiermacher unterschrieben und hinzugefügt, dass alle Kulturbereiche auch immer Sozialbeziehungen einschließen, die nicht den Einzelnen fremd gegenüberstehen. Denn wenn auch große Organisationen wie der Staat den Bürgern als fern und fremd erscheinen können, so leben wir doch auch in ganz anderen, kleineren sozialen Kontexten. In Liebe, Freundschaft, Kollegialität, Sozialdienst, ja in allen Formen solidarischen Handelns, trennt sich keine objektive Kultur als fremd von der subjektiven ab.

Was die Unterscheidung des Wichtigen und Unwichtigen angesichts der Informationsflut betrifft, so füge ich hier einen eigenen Gedanken an, der bei Schleiermacher allenfalls impliziert ist. Wir können einige Wissensbereiche getrost den Spezialisten überlassen, andere nicht. Wie man ein Auto repariert, muss nicht jedem gelehrt werden. Aber was moralisch oder unmoralisch ist, was für eine Demokratie schädlich und was nützlich ist, das sollte jeder verantwortliche Mensch und jeder Bürger wissen und immer bes-

7 Siehe die „Güterlehre“ in Schleiermachers philosophischer Ethik: Entwürfe zu einem System der Sittenlehre, hg. von Otto Braun, Schleiermachers Werke, Bd. 2 (Leipzig ${ }^{2} 1927$ ), S. 241-371, 421-455, 513-626. Ein Überblick über Begründung und Ordnung dieser Sozialsphären bei Gunter Scholtz: Ethik und Hermeneutik. Schleiermachers Grundlegung der Geisteswissenschaften, Frankfurt/Main 1995, S. 36 f.

${ }^{8}$ M. Walzer, Spheres of Justice: a defense of pluralism and equality, New York 1983. 
ser wissen wollen, ebenso, was Kunst ist und was nicht, und was Religion bedeutet. Die „Bildung“ gälte demnach vor allem den Bereichen, in denen jeder unvertretbar selbst entscheiden muss.

\section{The Issue of General Education from the Historical Perspective (Summary)}

The development of the term encyclopaedia is the mirror of the historical growth of knowledge and change of education. In the ancient Greek period, the name of artistic and musical education encyclopaedia became the title of collections of all human knowledge and finally resulted in a crisis today. By the philosophy of Georg Simmel, we can understand the historical changes as growing separation and alienation between the cultural systems of society on the one hand, and the education of single persons of the other. In the thinking of Wilhelm von Humboldt, Friedrich Schleiermacher, and also Georg Simmel, we find important advices to enable real education also in the scientific civilisation and to overcome the situation in which knowledge and sciences became strange for the life of single human beings.

Keywords: culture; encyclopaedia; fine art; general education; growth of knowledge; humanity; philosophy; sciences.

\section{Problem kształcenia ogólnego z perspektywy historycznej (Streszczenie)}

Przeobrażanie się terminu ,encyklopedia“ stanowi odzwierciedlenie historycznego postępu wiedzy i zmian w edukacji. W Starożytnej Grecji nazwa encyklopedii malarstwa i muzyki stała się tytułem zbiorów dotyczących całej ludzkiej wiedzy, co w rezultacie przyczyniło się do współczesnego kryzysu. Zgodnie z filozofią Georga Simmela, możemy rozumieć zmiany historyczne jako postępujący rozdział pomiędzy systemami kulturowymi społeczeństwa z jednej strony i kształcenia jednostek z drugiej. W filozofii Wilhelma von Humdoldta, Friedricha Schleiermachera, jak również Georga Simmela, odnajdujemy ważne wskazówki dotyczące możliwości prawdziwego kształcenia również w cywilizacji nauki i przezwyciężenia sytuacji, w której wiedza i nauka stają się obce dla przeciętnego człowieka w kontekście jego życia.

Słowa kluczowe: kultura; encyklopedia; sztuka; edukacja ogólna; przyrost wiedzy; humanizm; filozofia; nauki. 
